(C) [2005] IEEE. Reprinted, with permission, from [Moha'med Al-Jaafreh and Adel Al-Jumaily, Multi agent system for estimation of cardiovascular parameters, Computers, Communications, \& Signal Processing with Special Track on Biomedical Engineering, 2005. CCSP 2005. 1st International Conference on 14-16 Nov. 2005]. This material is posted here with permission of the IEEE. Such ermission of the IEEE does not in any way imply IEEE endorsement of any of the University of Technology, Sydney's products or services. Internal or personal use of this material is permitted. However, permission to reprint/republish this material for advertising or promotional purposes or for creating new collective works for resale or redistribution must be obtained from the IEEE by writing to pubs-permissions@ieee.org. By choosing to view this document, you agree to all provisions of the copyright laws protecting it 


\title{
Multi Agent System for Estimation of Cardiovascular Parameters
}

\author{
Moha'med Al-Jaafreh and Adel Al-Jumaily \\ Mechatronics and Intelligent Systems Group, Faculty of Engineering, University of \\ Technology, Sydney
}

\begin{abstract}
:
Many cardiovascular diseases can be avoided by continuous monitoring cardiovascular parameters. Heart rate, electrocardiogram, blood pressure and pulse wave velocity are the most important and popular cardiovascular parameters. These parameters can be measured by different sensors that have been developed and improved to achieve reliable, accurate and continuous measurements. A part of the processing of theses sensors data, to get the related information, is parameters estimation.

This paper presents a new concept to estimate cardiovascular parameters via using a new multi-agent system; that combines two independent methods; first method depends on pulse wave velocity (PWV), while second method depends on heart rate and artery resistance.

The outcome of this multi-agent system is a continuous and reliable estimation of cardiovascular parameters by using non-invasive, Cuffless cheap sensors.
\end{abstract}

\section{1- Introduction:}

The cardiovascular system consists of heart, vessels and blood. In healthy person; the heart pumps the blood in vessels with synchronous pulses (heart rate) and velocity PWV. The force of blood flux, which caused by heart beating, is formed a pressure against blood vessels' walls (blood pressure).

Many methods have been used to measure blood pressure that depended on blood level and blood density such as arterial cannulation; which was firstly used by Ludwig on1847, later it were be developed to be depended on applying external pressure and heart beat such as palpation and auscultatory; which were firstly used by Rocci in 1896 and Korotkoff in 1905, respectively (Beecher 2003), afterwards developed to be depended on photo-plethysmography (PPG) and artery volume changes such as volume-compensation and local pressurization, which were used by Penaz in 1973, then depended on piezoelectric pressure transducers such as tonometry (Mackay 1962), Finally depended on PPG electric-plethysmography (EPG) (Yang 2000, Cheang 2003).

On other hand, Blood pressure has been estimated depending on pulse arrival time and pulse wave velocity (Bazett 1922 and Lansdown 1957). In addition, Pulse wave velocity and pulse arrival time has been used to estimate blood pressure changes (Geddes 1981, Pitson 1998 and Chen 2000). Previous methods have achieved some of physicians and clinicians requirements and expectations with many limitations and disadvantages. Arterial cannulation method is the standard method; which has been used to measure a blood pressure, but it invasively since it used catheter; which is inserted into the artery. The second standard method is the auscultatory method; which estimates blood pressure discontinuously; since it depends on applying external pressure through cuff; which needs time to inflation or deflation. In addition, External pressure caused veins occlusion and discomfort for the person.

The recent research studies on that standard methods have attempted to decrease these limitations; Volumecompensation, local pressurization and tonometry methods reduce the external blood pressure affection. PPG and EPG offers new technology by measuring volume changes, artery diameter and pulse wave velocity through infra-red signals. Unfortunately, the measurements of these methods are under process to improve the reliability and accuracy.

This paper will present a new multi-agent system to estimate cardiovascular parameters. This model combines two independent methods; first method depends on PWV and second method depends on heart rate and artery resistance.

Section 2 of this paper, the Multi-agent model and the methods' equation will be explained, the Experiment of two sides; simulation and circuit model with their results are explained in section 3, and the work results will be discussed, summarized and concluded in section 4 .

\section{2- Multi-agent model:}

The Multi Agent system is a new technic depends in combining many agents to achieve one or more goals; if agents combined in an appropriate model; they will achieved progressive and better results with cheap tools. In this paper; the cardiovascular parameters will be estimated by using a multi-agent model; which is illustrated in figure (1). This model is consists of two agents (methods); heart rate and arterial resistance method and pulse wave velocity method. These two methods extract their elements from the PPG signal; which is measured by attaching a PPG sensor on index finger.

The estimation will be implemented by combing the two methods and by calculating the root mean square value of the two blood pressure values.

\section{1 - Pulse wave velocity Method:}

Our method to find the PWV is by using two PPGs sensors; which attached to subject's finger with distance $(\mathrm{t})$ between two sensors.

To estimate the blood pressure from PWV; the blood 


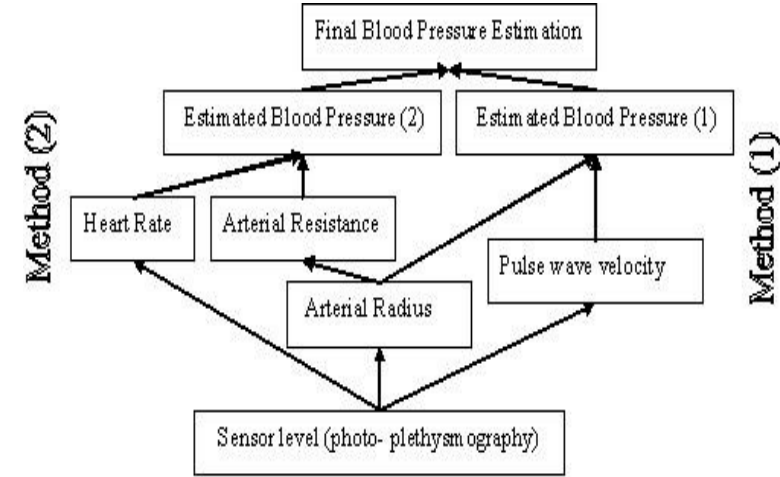

Fig (1) Block diagram of Multi agent model pressure is

related with vessel wall elastic modulus via equation (Hughes 1979):

$$
E=E_{0} e^{\gamma P}==>P=\cdot \frac{1}{\gamma} \ln \frac{E}{E_{0}}
$$

Where $\mathrm{P}$ is blood pressure

$\gamma$ is coefficient, changes with vessel type

$E_{0}$ is the artery elasticity when the blood pressure equal zero

$E$ is vessel wall elastic modulus

Consequently, the vessel wall elastic modulus is related with pulse wave velocity as shown in Moens-Korteweg formula (Chen 2000):

$$
v=\sqrt{-\frac{g E a}{\rho d}}==>\quad E=-v^{2} \rho d \text {-------- (2) }
$$

Where $v$ is pulse wave velocity

$\mathrm{g}$ is gravity acceleration

a is thickness of artery wall

$\rho$ is blood density

$d$ is artery diameter

Through equations (1) and (2); the blood pressure is estimated by substituting value of elasticity modulus (E) of equation (1) in equation (2). Therefore, the relationship between blood pressure and PWV becomes:

$$
P=\frac{1}{\gamma} \ln \left(\frac{v^{2} \rho d}{g a E_{0}}\right) \text {---------------- (3) }
$$

\section{2- Blood artery resistance Method:}

The Dynamic flow of blood in body, cardiovascular system, can be considered as an electric circuit (Carr 2001). Therefore, Ohms' law can be applied on the cardiovascular electric circuit.

$$
\text { Ohms' law: V = I x R }
$$

In the cardiovascular electric circuit; a difference of mean blood pressure $\left(\mathrm{P}_{\mathrm{M}}\right)$ is corresponding to voltage potential difference $(\mathrm{V})$, a blood flow (BF) within vessels is corresponding to current (I) and the total blood vessel's resistance (TR) against blood flow is corresponding to resistance (R). Therefore, as ohm's law, this formula is written:

$$
\boldsymbol{P}_{M}=\text { BF } x \text { TR }
$$

The blood flow can be calculated by measuring the volume of blood, which is pumped from heart per beat, this volume is equal to the difference of heart volume before (HVB) pumping blood and heart volume after (HVA) at that moment. This volume is called stroke volume (VS).

$$
\text { VS = HVB - HVA ----------- (6) }
$$

The heart pumps this amount of blood every heart beat and the $\mathrm{BF}$ is calculated by liter/min. Hence,

$$
\text { BF = VS x h ------------- (7) }
$$

Where $\mathbf{h}$ is number of heart beats/ minute Then, equation (5) becomes:

$$
P_{M}=h \times V S \times T R
$$

The blood vessels' resistance is a function of blood viscosity $(\eta)$, and inversely varies with number of vessels $(\mathrm{N})$ and radius of vessels $(\mathrm{r})$ :

$$
R=f(\eta / N, r)
$$

Hence, blood vessel is considered as cylindrical tube. Then, the resistance of blood vessels against blood flow can be calculated as (Jan 2004):

$$
T R=8 L \eta / N \pi r^{4}
$$

Where $L$ is the length of artery

The number of heart beats/minute (h); heart rate, is calculated from PPG signal by equation:

$$
h=60 \times f
$$

Where $\boldsymbol{f}$ is the frequency of PPG signal.

\section{3- Experiment:}

The multi-agent model have been executed in two procedures; firstly by simulation then by real measurements via using a model circuit of PPG sensor attached to index finger of person.

\section{1- Simulation Model:}

The previous equations have been simulated by considering heart rate (h) and PWV (v) as variables and considering other factors' as constants; the ranges of variable factors and the values of constant factors for healthy adult are offered from the references attached after each factor.

$\gamma=0.017 \mathrm{mmHg}^{-1}$ (Chen 2000, Hughes 1979)

$\rho=1035 \mathrm{Kg} / \mathrm{m}^{3}$ (Elert 1998-2005)

$\mathrm{g}=9.81 \mathrm{~m} / \mathrm{s}^{2}$ the acceleration of gravity.

$E_{0}=187500 \mathrm{~N} / \mathrm{m}^{2}$ (Hughes 1979).

VS $=70 \mathrm{~cm}^{3} \quad$ (Klabunde 2005)

$\eta=4.5$ m.Pa.s (Elert 1998-2005)

$\mathrm{L}, \mathrm{N}, d=2 r$ these values are different according to artery types; for these calculations the aorta's average values will be taken:

$\mathrm{L}=0.4 \mathrm{~m}, \mathrm{~N}=1, \mathrm{R}=0.0125 \mathrm{~m}$. (Gray's anatomy 1995)

$\boldsymbol{h}=40-120 \mathrm{beat} / \mathrm{min}$ (AHA 2005)

$v=3-13 \mathrm{~m} / \mathrm{s}$ (AHA 2005) 
The estimated Systolic blood pressure (SBP) by using PWV method has been shown in figure (2); which is shown the value of PWV $=7.75 \mathrm{~m} / \mathrm{s}$ faced normal SBP $=120 \mathrm{mmHg}$ that it is little bit higher than value of $\mathrm{PWV}=7.43 \mathrm{~m} / \mathrm{s}$; that average value of $\mathrm{PWV}$ has been found (Persson 2001).

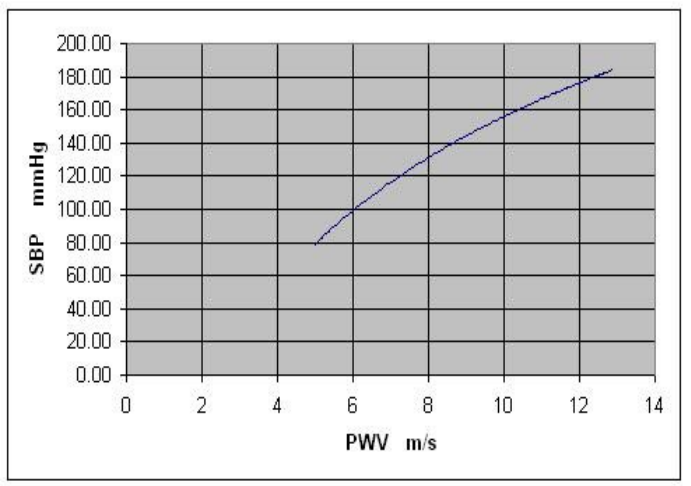

Fig (2) estimated blood pressure by PWV method

The estimated blood pressure by using arterial resistance method has been shown in figure (3); the heart rate variable is chosen to show the value of heart rate; which faced normal SBP (SBP $=120 \mathrm{mmHg}$ ) is $\boldsymbol{h}$ $=53.4 \mathrm{beat} / \mathrm{min}$ and it is little bit lower than average heart rate $60 \mathrm{beat} / \mathrm{min}$.

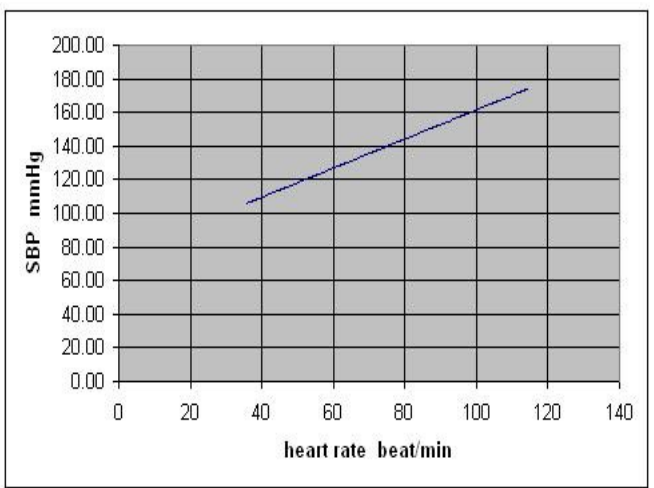

Fig (3) estimated blood pressure by arterial resistance method

Therefore, these two methods are combined by calculating the root mean square value of the two systolic blood pressures from two methods. That will present acceptable measurements of real systolic blood pressure as shown in figure (4).

\section{2- Circuit Model:}

This model of the circuit uses a photo-plethysmography sensor to measure PWV and heart rate. The PPG sensor consists of infrared light emitting diode (LED) as transmitter and a photo-diode as a receiver. Circuit model is shown in figure (5). The output of photo-diode is the PPG signal as shown in figure (6). A filter is used to remove DC level, the output of this circuit will be attached to a microcontroller to calculate artery resistance then estimate blood pressure through the previous equations.

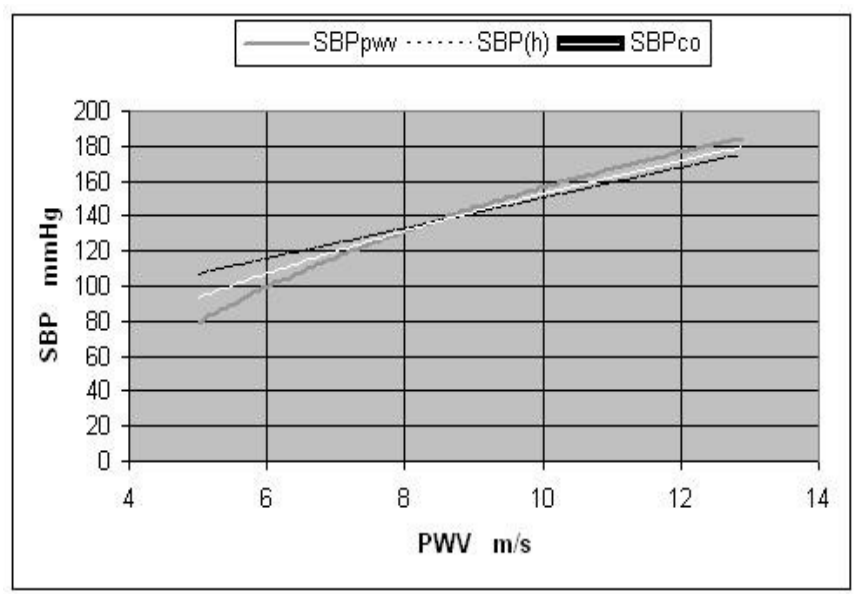

Fig (4) Final blood pressure estimation

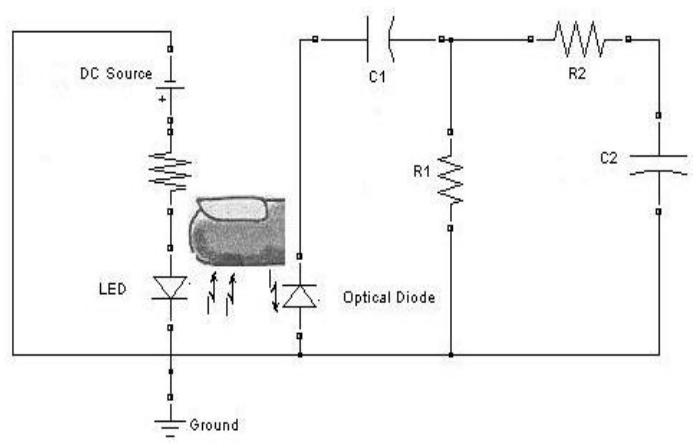

Fig (5) Circuit Model

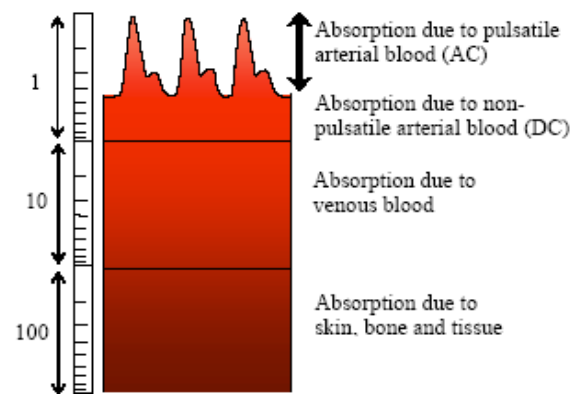

Fig (6) output of photodiode

\section{3- Results:}

The model circuit was attached to an adult healthy person's index finger, the output of that circuit was attached to oscilloscope as Figure (7) shown. The PPG signal is represent by the output signal of the circuit.

From PPG signal, the heart rate can be calculated. The frequency of PPG signal is equal:

$$
f=1 /(1.8 \times 500 \mathrm{~ms})=1.1 \mathrm{~Hz}
$$

Therefore, the heart rate $(\boldsymbol{h})$ by equation (11):

$$
\boldsymbol{h}=60 \times f=66 \mathrm{beat} / \mathrm{min}
$$

Then by using the constants' values; as mentioned in section 3.1 and equation (10), the value of Total arterial resistance is:

$\mathrm{TR}=8 \times 0.4 \times 4.5 \times 10^{-3} / \pi(0.0125)^{4}=187747 \mathrm{~Pa} . \mathrm{s} / \mathrm{m}^{3}$

Then value of Systolic blood pressure (SBD) is:

$$
\boldsymbol{P}=167.1 \mathrm{~Pa} \sim 125 \mathrm{mmHg}
$$




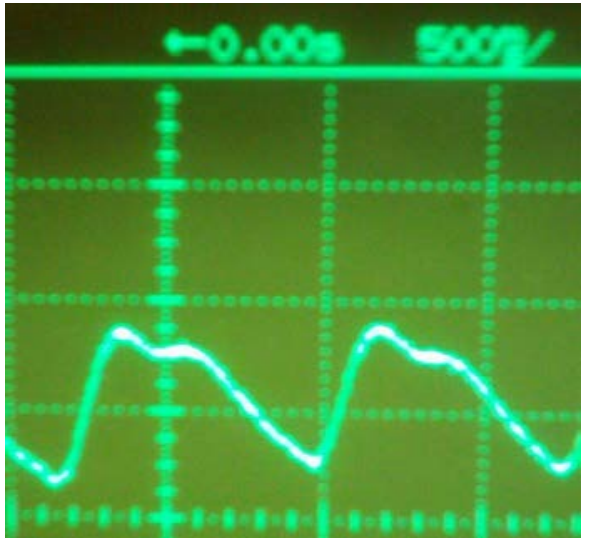

Fig (7) Output signal of Model circuit fig (5)

This outcome is within normal range for Adult's SBP (AHA); that prove the efficiency of this model.

\section{4- Discussion and Conclusions:}

These experiments clarify the conformity of implementation of one method; heart rate and arterial resistance method from both sides the simulation and real time measurements.

This paper presented a new method to measure a blood pressure by using two methods pulse wave velocity and artery resistance; these two methods are extract there factors from measured PPG signal.

This technique is applied multi-agent cooperation concepts by combined different methods to achieve many objectives. Using multi-agent cooperation reduces the error percentage and increases measurements accuracy. Moreover, some of physicians and clinicians expectations will be achieved; such as comfortable, continuous and accurate blood pressure measurements; since this model depend on PPG sensors and continuous processed estimation via two methods. The future work is to test the conformity of whole multi-agent model and to measure continuous, reliable Blood pressure, heart rate and other cardiovascular parameters.

\section{5- References:}

1. American heart association (AHA), 2005, Available on line at: http://www.americanheart.org/

2. Bazett, H. and Dreyer, N., 1922, "Measurement of pulse wave velocity', Am. J. Physiol., 63, pp. 94115.

3. Beecher et al., 2003, "Blood pressure Monitoring, Department of Medical Physics and Bioengineering, University College London.”, Available on line at:http://www.medphys.ucl.ac.uk/teaching/undergr ad/projects/2003/group_03/history.html, last accessed 5/2/2005.

4. Chen, W., Kobayashi, T., Ichikawa, S., Takeuchi, Y. and Togawa, T., 2000, "Continuous estimating of Systolic blood pressure using the pulse arrival time and intermittent calibration”, Med. Biol. Eng. Computer, vol. 38, pp.569-574.

5. Cheang, P. and Peter, R., 2003, "An Overview of Noncontact Photoplethysmography.” , Department of Electronic and Electrical Engineering, Loughborough University.

6. Elert G., 1998- 2005, "Physics Hypertext-book", Available on line at: http://hypertextbook.com/physics/, last accessed 5/2/2005.

7. Geddes, L., Voelz, M., Babbs, C., Bourland, J. and Tacker, W. A., 1981, "Pulse transit time as an indicator of arterial blood pressure”, Psychophysiology, 18, pp. 71-74.

8. Hughes, D., Babbs, C., Geddes, L. and Bourland, J., 1979, "Measurement of Young's modulus of elasticity of the canine aorta with ultrasound", Ultrasonic Imaging, 1, pp. 356-367.

9. Hast J., 2003, "Self-mixing interferometry and its applications in non-invasive pulse detection", University of Oulu, Finland.

10. Klabunde R., 2005, "Cardiovascular Physiology Concepts", Available on line at: http://cvphysiology.com/index.html , last accessed $5 / 2 / 2005$.

11. Lansdown, M., 1957, "A method of using induced waves to study pressure propagation in human arteries", Circ. Res., 5, pp. 594-601.

12. Mackay, R., 1964, "The application of physical transducer to intra-cavity measurement, with special application to tonometry”, Med. Electron. Biol. Eng., 2, pp. 3-17.

13. Penaz, J., 1973, "Photo-electric Measurement of blood pressure, Volume and Flow in the Finger", Digest of the 10-th Int. Conf. on Medical and Biol. Eng.

14. Persson M., Eriksson H. and Lindstrom K., 2001, "Estimation of arterial pulse wave velocity with a new tissue Doppler method", Proceeding $23^{\text {rd }}$ Annual International conference of IEEE Engineering in Medicine and Biology Society, Istanbul.

15. Piston, D. and Stradling, J., 1998, "Value of beat-tobeat blood pressure changes, detected by pulse transit time, in the management of the obstructive sleep apnoea/hypopnoea syndrome”, Europ. Respirat. J., 12, pp. 685-692.

16. Yang, B., Asada, H. and Zhang, Y., 2000, " Cuff-less Continuous monitoring of Beat-to-Beat blood pressure using a Kalman Filter and Sensor Fusion.”, MIT Home Automation and healthcare Consortium. 